JAOS

JOURNAL OF APPLIED ORAL SCIENCE

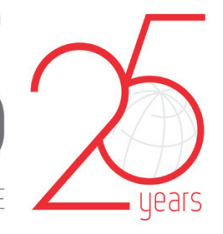

\title{
Treatment of experimental periodontal disease by laser therapy in simvastatin-modified rats
}

\section{Abstract}

Andressa Araújo SWERTS ${ }^{1}$

Bianca Fernanda Espósito SANTOS ${ }^{1}$

Simone Ribeiro BRUZADELLI ${ }^{1}$

Maísa Ribeiro Pereira Lima

BRIGAGÃO2

Daniela Coelho de LIMA ${ }^{1}$

Leandro Araújo FERNANDES ${ }^{1}$
Submitted: September 20, 2016 Modification: December 19, 2016

Accepted: December 29, 2016
Corresponding address: Leandro Araújo Fernandes

Departamento de Clínica e Cirurgia - Faculdade de Odontologia - Universidade Federal de Alfenas. Rua Gabriel Monteiro da Silva, 700 - Centro - Alfenas - MG - Brazil - 37130-000 - Phone: 55-35-3299-1000 e-mail: learaujofernandes@gmail.com
Low intensity laser can be used as a promising alternative in the treatment of periodontal disease. Objective: The aim of this study was to evaluate low-level laser therapy (LLLT) as an adjuvant treatment for scaling and root planing (SRP) for the treatment of induced periodontitis in simvastatinmodified rats. Material and Methods: A total of 180 rats were evenly divided into two groups: Veh - receiving oral administration of polyethylene glycol (vehicle); S - receiving oral administration of Simvastatin. Periodontal disease was induced in both groups at the first mandibular molar. After seven days, the ligature was removed and the animals were divided into subgroups according to the following local treatments: NT - no treatment; SRP - scaling and root planing and irrigation with saline solution; and LLLT $\neg-$ SRP and laser irradiation (660 nm; $0.03 \mathrm{~W} ; 4 \mathrm{~J}$ ). Ten animals in each subgroup/local treatment were euthanized at 7, 15 and 30 days. Samples of gingival tissue were processed to analyze the tissue oxidative damage and radiographic analysis. Levels of oxidative stress were analyzed by the expressions of Tripeptideglutathione (TG), Malondialdehyde (MDA) and Carbonylated Proteins (CP). Results: The animals in S group had higher levels of TG and lower levels of MDA and CP compared with Veh group ( $p<0.05)$. Radiographically, in the intragroup analysis Veh and S, LLLT showed lower bone loss (BL) compared with NT and SRP, in all experimental periods $(\mathrm{p}<0.01)$. In addition, a lower $\mathrm{BL}$ was observed for the animals of Veh group treated with LLLT compared with treatment SRP in the S group, in all experimental periods. Conclusion: Within the limits of this study, we can conclude that LLLT was effective as adjuvant treatment for SRP protecting against the occurrence of oxidative tissue damages as well as for reducing alveolar bone loss in experimentally induced periodontitis simvastatin-modified rats.

Keywords: Alveolar bone. Laser therapy. Oxidative stress. Periodontal diseases. Simvastatin.

${ }^{1}$ Universidade Federal de Alfenas, Faculdade de Odontologia, Departamento de Clínica e Cirurgia, Alfenas, MG, Brasil.

'Universidade Federal de Alfenas, Faculdade de Odontologia, Departamento de Bioquímica, Alfenas, MG, Brasil. 


\section{Introduction}

In inflammation, neutrophils are the first cells to be activated to defend the body together with macrophages ${ }^{27}$. Those are chemotaxically attracted by secretory cells, bacteria and other foreign bodies to inflammation areas ${ }^{27}$. On this site, neutrophils phagocyte the microorganisms, covered or not with complement or specific antibodies, which are killed by cytotoxic proteins derived from cytoplasmic granules and by oxygen and nitrogen reactive species such as superoxide anion ${ }^{9}$, hydrogen peroxide ${ }^{28}$, hydroxyl radical $^{12}$ and peroxynitrite ${ }^{21}$.

There is evidence of a more aggressive destruction of tooth support tissues with elevated levels of oxidative stress markers during the development of periodontal disease (PD) ${ }^{24}$. Among the oxidants, the superoxide anion ${ }^{9}$, in periodontal tissues, can be involved in signaling of induction of bone resorption; the hydroxyl radical ${ }^{12}$ is extremely reactive and can damage important biomolecules, such as proteins, lipids and nucleic acids, whereas hydrogen peroxide is able to cross membranes, damaging adjacent cells and increasing the oxidative cascade ${ }^{28}$. Thus, most studies demonstrate that periodontitis is associated with increased lipid peroxidation ${ }^{29}$ and increased protein carbonyls ${ }^{9}$ as well as decreased antioxidants, such as reduced glutathione $(\mathrm{GSH})^{11,29}$. In addition, there are evidences of decreased oxidative injuries and changes in antioxidant system following periodontal treatment ${ }^{19}$.

The treatment of PD is based on the elimination of pathogenic subgingival microbiota by scaling and root planing (SRP) ${ }^{24}$. However, mechanical therapy used alone may be defective in the elimination of pathogenic bacteria, since they are located within soft and hard tissues or in areas that are inaccessible to periodontal instruments ${ }^{7}$. In addition, an important component of individuals themselves can lead to tissue destruction observed in the periodontitis. Therefore, therapeutic strategies performing the pharmacological modulation of host response have emerged as a new therapeutic approach $^{13}$.

Simvastatin is an inhibitor of the 3-hydroxy-3methyl-glutaryl-coenzyme A (HMG - CoA reductase) enzyme, which is responsible for the synthesis of cholesterol and therefore it is widely used for the systemic treatment of diseases related to hypercholesterolemia. This drug also has antiinflammatory, immunomodulator, antioxidant, and angiogenic effects ${ }^{6,25}$, and it also promotes increased osteoblast formation ${ }^{10,20}$. Such properties offer great potential for statins to modify the course of chronic inflammatory diseases such as periodontitis ${ }^{26}$.

In addition to drug therapy, the use of low intensity lasers associated with scaling and root planing for the local treatment of periodontal disease has been reported ${ }^{7}$. On the other hand, there are no studies evaluating the local effects of low-level laser therapy (LLLT) associated with the systemic effects of statins. Thus, the aim of this study was to evaluate LLLT as an adjuvant treatment for scaling and root planing (SRP) in the treatment of induced periodontitis in simvastatin-modified rats.

\section{Material and methods}

Animals

This study was conducted on 180 adult male Wistar rats (200-250 g). The animals were kept in plastic cages with access to food and water ad libitum. Prior to surgical procedures, all animals were allowed to acclimatize to the laboratory environment for a period of five days. All protocols described below were approved by the Ethics Committee on the Use of Animals (CEUA), following the standards adopted by the Brazilian College of Animal Experimentation (COBEA), under protocol 472/2012.

\section{Study design}

Animals were numbered and randomly divided into two groups: Veh group $(n=90)$ received Polyethylene Glycol 400 (All Chemistry; São Paulo, SP, Brazil) at $0.5 \mathrm{mg} / \mathrm{kg}$ body weight (vehicle), and $\mathrm{S}$ group $(\mathrm{n}=90)$ received Simvastatin (Medley; Campinas, SP, Brazil) at $0.5 \mathrm{mg} / \mathrm{kg}$ body weight orally ${ }^{18}$. Administrations were daily performed in a single dose, starting $24 \mathrm{~h}$ before induction of PD and maintained until the end of the respective periods of euthanasia. Animals were weekly weighed throughout the experimental period to maintain the doses.

Simvastatin preparation was performed by diluting $400 \mathrm{mg}$ of the drug in $400 \mathrm{mlL}$ of Polyethylene Glycol to reach a final concentration of $1 \mathrm{mg} / \mathrm{mL}$ (Moss Manipulation Pharmacy; Alfenas, MG, Brazil).

Induction of experimental periodontal disease General anesthesia was induced by administering 
ketamine $(0.4 \mathrm{~mL} / \mathrm{kg}$ ) (Fort Dodge Animal Health Ltda; Campinas, SP, Brazil) together with xylazine (0.2 mL/kg) (Coopers; São Paulo, SP, Brazil) via intramuscular injection. The mandibular left first molar from each animal in both Veh and $\mathrm{S}$ groups was selected to receive a cotton ligature No. 10 (Coats; São Paulo, SP, Brazil) in a submarginal position to induce experimental periodontitis ${ }^{2}$.

\section{Local treatment}

After seven days of periodontal disease experimental induction, mandibular ligature was removed from the left first molar of all animals in groups Veh and $\mathrm{S}$. The animals were divided into subgroups according to the local treatments (performed only once): NT - no treatment; SRP - scaling and root planing and irrigation with saline solution; and LLLT - SRP and laser irradiation.

Left molars were subjected to SRP with manual \#1-2 micro mini five curettes (Hu-Friedy; Chicago, IL, USA) through 10 distal-mesial traction movements in both buccal and lingual aspects. The furcation and interproximal areas were scaled with the same curettes through cervical-occlusal traction movements. The entire SRP procedure was performed by the same experienced operator. The saline solutions were slowly deposited within the periodontal pocket using syringe $(1 \mathrm{~mL})$ and insulin needle $(13 \mathrm{~mm} \times 0.04 \mathrm{~mm}$ ) (Becton Dickinson; Curitiba, PR, Brazil).

The laser used in this study was galliumaluminium-arsenide (GaAlAs) (Kondortech Equipment Ltd; São Carlos, SP, Brazil), with a wavelength of $660 \mathrm{~nm}$ and a spot size of $0.07 \mathrm{~cm}^{2}$. After $1 \mathrm{~min}$ of saline solution application, LLLT was applied to three equidistant points at each buccal and lingual aspect of the mandibular first molar in contact with the tissue. The therapeutic laser was released with a power of $0.03 \mathrm{~W}$ for $133 \mathrm{~s} /$ point, a power density of $0.428 \mathrm{~W} /$ $\mathrm{cm}^{2}$, and energy of $4 \mathrm{~J} /$ point $\left(57.14 \mathrm{~J} / \mathrm{cm}^{2} / \text { point }\right)^{7}$.

\section{Experimental periods}

Ten animals from each experimental subgroup/local treatment were euthanized by exsanguination at 7, 15 and 30 days following local treatments. The jaws were removed and, in order to analyze the levels of oxidative stress, collections of the inserted and marginal gums of the buccal faces of the first left lower molars were performed. Then, the jaws were split in the middle and fixed in $10 \%$ buffered formalin for at least $48 \mathrm{~h}$ for later radiographic analysis of the left side.

\section{Preparation of gingival samples}

Gingival tissue samples were homogenized with phosphate buffer ( $3 \mathrm{~mL}, \mathrm{pH} 6.5$ ). The homogenate was centrifuged at $3500 \mathrm{rpm}$ for $10 \mathrm{~min}$. The supernatant was separated into aliquots for subsequent biochemical analysis.

\section{Determination of the total protein concentration}

Protein concentrations were determined in all samples of gingival homogenates by the method of Bradford ${ }^{4}$, using bovine serum albumin (BSA) as a standard calibration curve. The total protein concentration is routinely determined for normalization of biochemical results. Thus, the expression results in the mass in $\mathrm{mg}$ of tripeptideglutathione (TG), malondialdehyde (MDA) and carbonylated proteins (CP) per mg protein (units).

\section{Determination of tripeptideglutathione ( $T G$ )}

For verification of TG in the gingival tissue of rats alkylated derivatives of $\mathrm{mBBr}$ were separated by HPLC in C-18 column (Shim-pack VP-ODS, $4.6 \mathrm{~mm} \times 25 \mathrm{~cm}$, $5 \mu \mathrm{m}$, connected in series with C-18 precolumn model Shim-pack GVP-ODS, $4.6 \mathrm{~mm} \times 10 \mathrm{~mm}$ ), equilibrated with buffer $A(14.2 \%$ ethanol and $0.25 \%$ acetic acid $\mathrm{m} / \mathrm{v}, 1 \mathrm{~mL} / \mathrm{min})$. Samples of tissue suspensions derivatized with $\mathrm{mBBr}$ were injected and dilution (buffer A) was performed for $30 \mathrm{~min}$. Then, the column was washed with buffer B (90\% methanol and 0.25\% acetic acid) for $8 \mathrm{~min}$ and re-equilibrated with buffer A. Sulfosalicylic acid (internal reference standard), cysteine and reduced glutathione were determined by comparing the retention time of authentic standards $(10-200 \mathrm{nmol})$ using a fluorescence detector (model $\mathrm{RF}-10 A X \mathrm{~L})$ and $\lambda$ exc $=394 \mathrm{~nm}$ and $\lambda \mathrm{emi}=490 \mathrm{~nm}(\varepsilon=20$ $\left.\mathrm{mM}^{-1} \cdot \mathrm{cm}^{-1}\right)$, and the quantification was performed by counting the units in the respective areas (Software "LC-Solution Multi") ${ }^{14}$.

\section{Determination of malondialdehyde (MDA)}

The MDA verification through homogenized gum samples was performed by adding $250 \mu \mathrm{L}$ of $1.22 \mathrm{M}$ phosphoric acid, $450 \mu \mathrm{L}$ of Milli-Q Water and $250 \mu \mathrm{L}$ of TBA reagent. Thereafter, aliquots are stirred for $30 \mathrm{~s}$ and the reaction is incubated for $1 \mathrm{~h}$ in water bath at $95^{\circ} \mathrm{C}$ and cooled in ice bath at $4^{\circ} \mathrm{C}$. After this process, $360 \mu \mathrm{L}$ of methanol (HPLC grade) and $40 \mu \mathrm{L}$ 
of $1 \mathrm{M} \mathrm{NaOH}$ are added to a $200 \mu \mathrm{L}$ sample, in order to neutralize the solutes and precipitate proteins before injecting into the HPLC column, using a fluorescence detector (model RF-10AXL) and $\lambda$ exc $=515 \mathrm{~nm}$ and $\lambda e m i=553 \mathrm{~nm}^{14}$.

\section{Determination of carbonylated proteins (CP)}

In order to determine these $C P, 500 \mu \mathrm{L}$ of the aliquots in phosphate buffer ( $\mathrm{pH}$ 7.2) may be used, adding $500 \mu \mathrm{L}$ of 2.4-dinitrophenylhydrazine solution $(10 \mathrm{m \mu})$. After that, incubate for $1 \mathrm{~h}$ and then apply (drops) $500 \mu \mathrm{L}$ of $20 \%$ TCA solution until complete precipitation. Add $500 \mu \mathrm{L}$ of Ethanol/Ethyl-Acetate solution and centrifuge at $2000 \mathrm{rpm}$ for $10 \mathrm{~min}$. Discard the supernatant and re-add $500 \mu \mathrm{L}$ of ethanol/ethyl-acetate solution and centrifuge it. Then, discard the supernatant and dissolve the precipitate with $1000 \mu \mathrm{L}$ of $6 \mathrm{M}$ guanidine, which should be homogenized. The rate of change in absorbance was spectrophotometrically measured at $370 \mathrm{~nm}^{14}$.

\section{Radiograph and digital analysis}

After fixation of the hemimandibules (HMs) in 10\% buffered formalin, the left side was submitted to X-ray procedure.

HMs were positioned on a table with the vestibular surfaces facing the radiographic film (Eastman Kodak Company; Rochester, Nova York, EUA), in such a way that the right side stayed at the bottom, and the left side stayed at the top.

Standardization of radiographs was obtained as follows:

Use of an X-ray device Pampas - E (CDK X-ray Equipment; Diadema, SP, Brazil), with electric system of $65 \mathrm{kvp}, 10 \mathrm{~mA}$;

Central X-ray beam perpendicular to the film-object plane, at a 90-degree angle in relation to the surface of the optical plate;

Focal length of $30 \mathrm{~cm}$;

Exposure time of 0.8 seconds.

Radiographs were developed using solutions from Kodak developer and fixer, using the climate-weather development method.

They were scanned and the images were analyzed with the Imagelab software (Softium; São Paulo, SP, Brazil) using the tool distance and angle of measurement. With this feature, it was measured the distance from the cementoenamel junction to the alveolar bone crest on the mesial surface of the first left lower molars by drawing a line, and these measurements were recorded in millimeters $(\mathrm{mm})$. The mouse was positioned on the region corresponding to the cementoenamel junction. By left-clicking and dragging the mouse down to the level of alveolar bone crest the software automatically measured the distance.

\section{Statistical analysis}

Statistical analysis of oxidative stress and radiographic data was performed by BioEstat 3.0 software (Sonopress; Manaus, AM, Brazil). The hypothesis of absence of a statistically significant difference in the data obtained in the region of the mandibular first molars between different groups, subgroups/treatments and periods in the teeth with induced periodontitis was tested. After the analysis of the normality of the data by the Shapiro-Wilk test, multiple comparisons among the variables were performed by two-way analysis of variance ANOVA with supplementation by the Tukey test, with $p<0.05$ (oxidative stress data) and by the Bonferroni test, with $\mathrm{p}<0.01$ (radiographic data).

\section{Results}

\section{Expression of TG in gingival samples}

In the intragroup comparison (Veh and S), TG levels increased significantly $(p<0.05)$ between the experimental periods in all local treatments. Regarding local treatments, LLLT showed a significant increase $(p<0.05)$ in GSH compared with NT and SRP at 7 and 30 days.

In the comparison between the groups (Veh and S), among the same local treatments, TG levels in the $S$ group were significantly higher than those in the Veh group $(p<0.05)$, for NT, SRP and LLLT in all experimental periods (Table 1 ).

\section{Expression of MDA in gingival samples}

In the intragroup comparison, levels of MDA in LLLT decreased significantly $(p<0.05)$ compared with NT, in the $\mathrm{S}$ group in all experimental periods, and in the Veh group at 7 and 30 days.

In the comparison between the groups (Veh and S), among the same local treatments, MDA levels in S group were significantly lower than those in Veh group $(p<0.05)$, for NT, SRP and LLLT at 7 days (Table 2 ). 
Table 1- Means and standard deviations $(M \pm S D)$ expression in TG units in the gingival tissue of the first lower left molar, according to each group, local treatment and period

\begin{tabular}{|c|c|c|c|}
\hline Group & & Veh & \\
\hline Periods & 7 days & 15 days & 30 days \\
\hline \multicolumn{4}{|c|}{ Treatments } \\
\hline NT & $5.02 \pm 0.24 \#^{* \prime 0}$ & $10.99 \pm 0.30 \#^{\circ}$ & $22.17 \pm 1.21 \#^{*^{\prime \prime}}$ \\
\hline SRP & $8.52 \pm 1.00 \#^{*^{\prime \circ}}$ & $15.01 \pm 0.30 \#^{\prime \circ}$ & $40.76 \pm 1.98 \#^{*^{\prime \prime}}$ \\
\hline LLLT & $10.13 \pm 1.13 \#^{* \prime \circ}$ & $16.07 \pm 1.64 \#^{\circ}$ & $52.49 \pm 0.58 \#^{*^{\prime}}$ \\
\hline Group & \multicolumn{3}{|c|}{$\mathrm{S}$} \\
\hline Periods & 7 days & 15 days & 30 days \\
\hline \multicolumn{4}{|c|}{ Treatments } \\
\hline NT & $14.15 \pm 0.34 \#^{* \prime \circ}$ & $23.11 \pm 1.67 \#^{\circ}$ & $43.87 \pm 0.56 \#^{\star^{\prime \circ}}$ \\
\hline SRP & $17.43 \pm 0.02 \#^{* \circ \circ}$ & $26.79 \pm 1.02 \#^{\circ}$ & $54.97 \pm 1.02 \#^{*^{\prime \circ}}$ \\
\hline LLLT & $23.29 \pm 0.17 \#^{* \circ \circ}$ & $24.32 \pm 1.09 \#^{\circ}$ & $64.98 \pm 1.12 \#^{*^{\prime \prime}}$ \\
\hline $\mathrm{N}$ & 60 & 60 & 60 \\
\hline
\end{tabular}

\#Difference among same groups and local treatments (ANOVA and $p<0.05$ )

*Difference between local treatments, same group and period (ANOVA and Tukey, $p<0.05$ )

Difference between groups, same local treatment and period (ANOVA and Tukey, $p<0.05$ )

${ }^{\circ}$ Difference between groups and local treatments, same period (ANOVA and Tukey, $p<0.05$ )

Table 2- Means and standard deviations $(M \pm S D)$ expression in MDA units in the gingival tissue of the first lower left molar, according to each group, local treatment and period

\begin{tabular}{|c|c|c|c|}
\hline Group & & Veh & \\
\hline Periods & 7 days & 15 days & 30 days \\
\hline \multicolumn{4}{|c|}{ Treatments } \\
\hline NT & $0.18 \pm 0.06^{\circ \circ}$ & $0.24 \pm 0.12^{\circ}$ & $0.30 \pm 0.03^{\circ}$ \\
\hline SRP & $0.28 \pm 0.12^{\circ 0}$ & $0.40 \pm 0.09$ & $0.62 \pm 0.13^{*}$ \\
\hline LLLT & $0.48 \pm 0.32^{* \prime 0}$ & $0.47 \pm 0.43^{*}$ & $0.34 \pm 0.17^{\circ}$ \\
\hline Group & & $S$ & \\
\hline Periods & 7 days & 15 days & 30 days \\
\hline \multicolumn{4}{|c|}{ Treatments } \\
\hline NT & $0.34 \pm 0.07^{\circ \circ}$ & $0.36 \pm 0.16$ & $0.44 \pm 0.08^{\circ}$ \\
\hline SRP & $0.53 \pm 0.22^{\circ}$ & $0.38 \pm 0.65$ & $0.61 \pm 0.73^{* \circ}$ \\
\hline LLLT & $0.72 \pm 0.54^{* \prime \circ}$ & $0.44 \pm 0.33^{* \circ}$ & $0.41 \pm 0.98^{\circ}$ \\
\hline$N$ & 60 & 60 & 60 \\
\hline
\end{tabular}

\#Difference among same groups and local treatments (ANOVA and $p<0.05$ )

*Difference between local treatments, same group and period (ANOVA and Tukey, $p<0.05$ )

'Difference between groups, same local treatment and period (ANOVA and Tukey, $p<0.05$ )

${ }^{\circ}$ Difference between groups and local treatments, same period (ANOVA and Tukey, $p<0.05$ )

Table 3- Means and standard deviations $(\mathrm{M} \pm \mathrm{SD}$ ) expression in CP units in the gingival tissue of the first lower left molar, according to each group, local treatment and period

\begin{tabular}{|c|c|c|c|}
\hline Group & & Veh & \\
\hline Periods & 7 days & 15 days & 30 days \\
\hline \multicolumn{4}{|c|}{ Treatments } \\
\hline NT & $13.23 \pm 0.31+^{* \prime \circ}$ & $8.45 \pm 1.56+^{*}$ & $7.89 \pm 1.95^{\star \circ}$ \\
\hline SRP & $7.02 \pm 0.31+^{* \prime o}$ & $2.39 \pm 1.13+^{* \circ}$ & $3.92 \pm 0.06+^{* \circ}$ \\
\hline LLLT & $4.71 \pm 0.23+^{* \prime o}$ & $1.99 \pm 0.94+^{* \circ}$ & $2.37 \pm 0.18+^{* \circ}$ \\
\hline Group & & $\mathrm{S}$ & \\
\hline Periods & 7 days & 15 days & 30 days \\
\hline \multicolumn{4}{|c|}{ Treatments } \\
\hline NT & $9.78 \pm .3+^{*^{\prime \circ}}$ & $7.99 \pm 11.3+^{* \circ}$ & $5.21 \pm 0.04+^{* \circ}$ \\
\hline SRP & $1.29 \pm 1.16+^{* \prime 0}$ & $4.80 \pm 1.12+^{* \circ}$ & $3.92 \pm 1.00+^{* \circ}$ \\
\hline LLLT & $1.57 \pm 0.95+^{* \prime 0}$ & $3.41 \pm 1.04+^{* \circ}$ & $1.09 \pm 0.32 t^{* \circ}$ \\
\hline $\mathrm{N}$ & 60 & 60 & 60 \\
\hline
\end{tabular}

\#Difference among same groups and local treatments (ANOVA and $p<0.05$ )

*Difference between local treatments, same group and period (ANOVA and Tukey, $p<0.05$ )

'Difference between groups, same local treatment and period (ANOVA and Tukey, $p<0.05$ )

${ }^{\circ}$ Difference between groups and local treatments, same period (ANOVA and Tukey, $p<0.05$ ) 
Table 4- Means and standard deviations $(\mathrm{M} \pm \mathrm{SD})$ of the distances between the cementoenamel junction and alveolar bone crest ( $\mathrm{mm}$ ) on the mesial surface of the first lower left molar, according to each group, local treatment and period

\begin{tabular}{|c|c|c|c|}
\hline Group & & Veh & \\
\hline Periods & 7 days & 15 days & 30 days \\
\hline \multicolumn{4}{|c|}{ Treatments } \\
\hline NT & $2.97 \pm 0.21+^{* \circ}$ & $2.53 \pm 0.34+^{* 0}$ & $2.06 \pm 0.13+^{* \circ}$ \\
\hline SRP & $1.88 \pm 0.31+^{* / 0}$ & $1.83 \pm 1.06^{* \circ}$ & $1.51 \pm 0.19+* \circ$ \\
\hline LLLT & $0.63 \pm 1.17^{\star \circ}$ & $0.58 \pm 0.93^{* \circ}$ & $0.49 \pm 1.34^{* \circ}$ \\
\hline Group & & $S$ & \\
\hline Periods & 7 days & 15 days & 30 days \\
\hline \multicolumn{4}{|c|}{ Treatments } \\
\hline NT & $2.65 \pm 0.33+{ }^{* \circ}$ & $2.37 \pm 0.43+^{* \circ}$ & $1.74 \pm 0.97+^{* \circ}$ \\
\hline SRP & $1.61 \pm 0.45^{\star \prime \circ}$ & $1.49 \pm 0.56^{* \circ}$ & $1.36 \pm 1.31^{* \circ}$ \\
\hline LLLT & $0.57 \pm 0.78^{* \circ}$ & $0.50 \pm 0.55^{\star \circ}$ & $0.47 \pm 0.88^{* \circ}$ \\
\hline $\mathrm{N}$ & 60 & 60 & 60 \\
\hline
\end{tabular}

\#Difference among periods, same groups and local treatments (ANOVA and Bonferroni, $p<0.01$ )

*Difference between local treatments, same group and period (ANOVA and Bonferroni, $p<0.01$ )

'Difference between groups, same local treatment and period (ANOVA and Bonferroni, $p<0.01$ )

${ }^{\circ}$ Difference between groups and local treatments, same period (ANOVA and Bonferroni, $p<0.01$ )
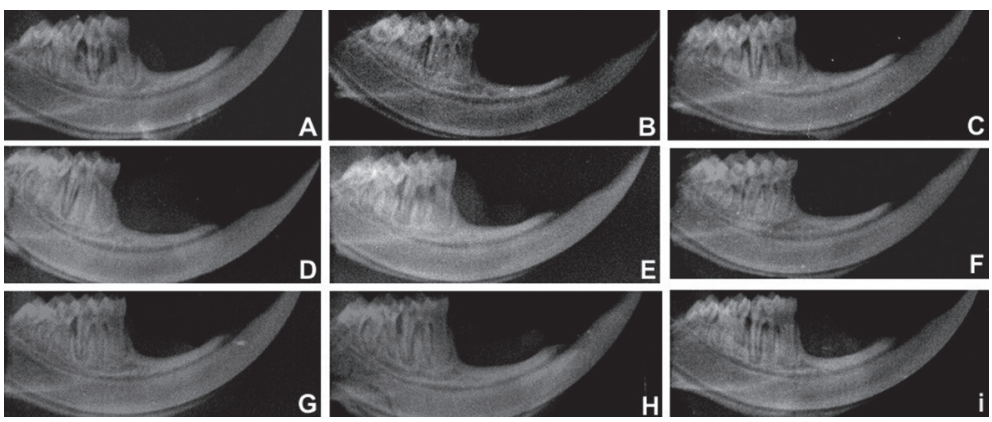

Figure 1- Radiographic images of the hemimandibules of animals of the Veh group -A: NT 7 days; B: NT 15 days; C: NT 30 days; D: SRP 7 days; E: SRP 15 days; F: SRP 30 days; G: LLLT 7 days; H: LLLT 15 days; and I: LLLT 30 days
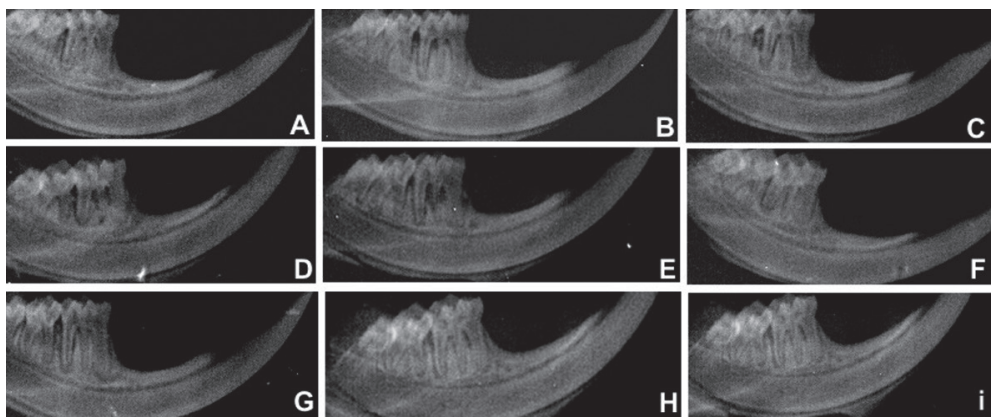

Figure 2- Radiographic images of the hemimandibules of animals of the S group - A: NT 7 days; B: NT 15 days; C: NT 30 days; D: SRP 7 days; E: SRP 15 days; F: SRP 30 days; G: LLLT 7 days; H: LLLT 15 days; and I: LLLT 30 days

\section{Expression of CP in gingival samples}

In the intragroup comparison (Veh and $\mathrm{S}$ ), levels of CP in LLLT decreased significantly $(p<0.05)$ in relation to NT in all the experimental periods.

In the comparison between Veh and $\mathrm{S}$ groups, among the same local treatments, CP levels in the S group were significantly lower than those in the Veh group $(p<0.05)$, for NT, SRP and LLLT at 7 days. In addition, CP levels in the Veh group, treated by LLLT, were significantly lower than those in the $\mathrm{S}$ group $(p<0.05)$, which did not receive local treatment (NT) at 7 and 15 days (Table 3 ).

\section{Radiographic analysis}

In the intragroup analysis between Veh and $\mathrm{S}$, LLLT showed lower BL compared with NT and SRP, in all experimental periods $(p<0.01)$.

In the intergroup analysis between the same local treatments, the SRP presented a lower BL $(p<0.01)$ in 
the animals of the $\mathrm{S}$ group $(1.61 \pm 0.45 \mathrm{~mm})$ compared with those in the Veh group $(1.88 \pm 0.31 \mathrm{~mm})$ in the 7-day period.

In the $\mathrm{S}$ group, LLLT values $(0.57 \pm 0.78 \mathrm{~mm}$, $0.50 \pm 0.55 \mathrm{~mm}, 0.47 \pm 0.88 \mathrm{~mm}$ ) showed lower $\mathrm{BL}$ $(p<0.01)$ compared with animals of the Veh group treated with SRP $(1.88 \pm 0.31 \mathrm{~mm}, 1.83 \pm 1.06 \mathrm{~mm}$, $1.51 \pm 0.19 \mathrm{~mm}$ ) in all experimental periods. In addition, a lower BL was observed for the animals of the Veh group treated with LLLT $(0.63 \pm 1.17 \mathrm{~mm}$; $0.58 \pm 0.93 \mathrm{~mm} ; 0.49 \pm 1.34 \mathrm{~mm}$ ) compared with SRP treatment in the $S$ group $(1.61 \pm 0.45 \mathrm{~mm} ; 1.49 \pm 0.56$ $\mathrm{mm} ; 1.36 \pm 1.31 \mathrm{~mm}$ ), in all experimental periods (Table 4, Figure 1 and Figure 2).

\section{Discussion}

Periodontal disease is a chronic inflammatory process characterized by gingival bleeding, formation of periodontal pockets and destruction of periodontal supporting tissues, through the release of lipopolysaccharide and proteases ${ }^{27}$ of bacteria present in the dental biofilm, a key etiological factor of this change. This tissue inflammation is associated with increased release of oxygen reactive species (ORS) by neutrophils, and also with the activation of several inflammatory mediators, such as interleukins (IL-1 $1 \beta$, 6 and 8 ) and tumor necrosis factor (TNF-a) ${ }^{11}$, which promotes the imbalance in bone homeostasis, resulting in destruction of alveolar bone tissue, increased activity of matrix metalloproteinases and connective tissue degradation ${ }^{17}$, due to exacerbated immune response and localized osteoclastogenesis ${ }^{12}$.

Periodontal treatment is used to paralyze the destruction of the supporting tissues of the teeth in order to avoid their loss ${ }^{5}$. However, there are cases where isolated periodontal mechanical therapy is ineffective, suggesting that systemic factors, not yet understood, could interfere with the development of the disease ${ }^{22}$. Thus, the use of antioxidants could be an adjuvant therapy to conventional treatment ${ }^{17}$.

Simvastatin is a drug with hypolipidemic function, but it stands out for other minor effects, including antiinflammatory ${ }^{25}$, immunomodulatory, and antioxidant effects, and the promotion of angiogenesis and increased differentiation of osteoblasts, inducing bone formation ${ }^{10,20}$. Such properties offer great potential for statin to modify the course of chronic inflammatory diseases ${ }^{26}$ such as chronic periodontitis.

Ligature-induced alveolar bone loss may occur due to abnormal activation of the host's immune system ${ }^{27}$. This will result in an imbalance, leading to an excessive production of oxidants and inhibiting the formation of antioxidants, which will cause the development of oxidative stress ${ }^{11}$. The association between systemic oxidative stress and periodontal disease in human and animal studies has been described in a review of the literature by Tomofuji, et al. ${ }^{28}$ (2011). This author mentioned that there is a correlation between the production of oxidants in sites with periodontal disease and the development of lesions in various organs of the body.

In animals, the association between ORS/ nitrogen reactive species (NRS) in sites of induced periodontitis is well established. With the use of an experimental model induced by topical application of lipopolysaccharide and specific proteases to the gums, Tomofuji, et al. ${ }^{28}$ (2011) showed a clear correlation between the severity of periodontal disease and oxidative lesions in the liver tissues and descending aorta. This oxidative stress can be evaluated in several ways such as by measuring the ORS levels, damages to nucleic acids, proteins and lipids, and detecting the levels of antioxidants ${ }^{3}$.

In this context, the proposed experimental model evaluated the concentration of TG, an endogenous antioxidant, and our results demonstrated that in both groups (Veh and S) NT showed significantly reduced levels compared with SRP and LLLT. This fact demonstrated that the development of oxidative damage occurred as well as in another study described in the literature using experimental periodontitis in rats $^{11}$.

Another widely used method for determining the occurrence of oxidative damage mediated by ORS in tissues is the measurement of MDA $^{3}$. Studies have demonstrated an association between periodontitis and increased MDA in samples of gingival fluid, saliva and gingival tissue ${ }^{11,28}$. In the intragroup comparison we observed that levels of MDA in NT were significantly higher $(p<0.05)$ compared with LLLT, in the $S$ group in all experimental periods, and in the Veh group at 7 and 30 days. These results demonstrate that MDA is a good marker of oxidative stress. Our results corroborate other studies ${ }^{11,23}$, which report that lipid peroxidation is a very common example of oxidative stress in induced periodontitis and that its increase plays an important 
role in the progression of periodontal destruction ${ }^{3}$.

The CP analysis is another way to verify the presence of oxidative tissue damage with changes mediated by inflammatory processes. In this model we observed a significant decrease in CP in the LLLT in the $S$ group compared with NT and SRP in Veh group $(p<0.05)$. This is probably due to the antiinflammatory effects of LLLT and simvastatin. A study carried out in $2003^{16}$ reinforced this hypothesis, since it demonstrated that simvastatin was able to inhibit the secretion of matrix metalloproteinases. Thus, they could reduce the inflammatory response, providing protection against the destruction of periodontal tissue. In humans, some studies have established that chronic periodontitis is directly correlated with the high occurrence of oxidative damage to proteins, determined by the measurement of serum CP as well as the increase in total oxidant status and lipid peroxidation products, quantified as $\mathrm{MDA}^{15,30}$. Whereas regarding LLLT, it was observed that it inhibited the production of inflammatory mediators ${ }^{1}$.

In the intragroup analysis between Veh and $\mathrm{S}$ groups, LLLT showed lower BL compared with NT and SRP in all experimental periods $(p<0.01)$. These results demonstrate that SRP was not effective in controlling bone loss in the furcation areas of animals. Clinically, it is proved that SRP with hand tools provides the best results for the treatment of periodontal disease. However, several factors may limit the success of SRP such as root concavities, dental crowding, deep areas, and areas of bifurcation that hinder the access of hand tools in the periodontal pocket. Due to these limiting anatomic factors, therapies to support conventional treatment have been proposed ${ }^{15}$.

Therefore, in this study simvastatin was chosen, since statins have different effects on bone, such as increased bone formation, whereas lovastatin and pravastatin have a smaller effect than simvastatin, atorvastatin and cerivastatin ${ }^{10}$. In addition, it stands out for acting in important events during an exacerbated inflammatory response. In the intergroup analysis between the same local treatments, the SRP presented a lower $B L(p<0.01)$ in the animals of the $S$ group compared with those in Veh group in the 7-day period. In S group, LLLT values showed lower $B L(p<0.01)$ compared with animals of Veh group treated with SRP in all experimental periods. This result is in agreement with other studies ${ }^{3,10,15}$. According to Luan, et al. ${ }^{16}$ (2003) statins decrease the production of many pro-inflammatory cytokines and have also been described as promoting decreased secretion of MMP-1 (matrix metalloproteinase - 1), MMP-2, MMP-3 and MMP-9 in vitro. Thus, they could reduce strong immune response, protecting against the destruction of periodontal tissue.

In addition, a lower BL was observed for the animals of the Veh group treated with LLLT compared with SRP treatment in the S group, in all experimental periods, confirming previous studies ${ }^{5,7}$ that demonstrate a better outcome of periodontal treatment with this combination, by stimulating bone formation 7,8,22. These studies have reported that the use of this light source inhibits the production of inflammatory mediators by cells of the periodontal ligament, promotes cell chemotaxis, and promotes local angiogenesis and vasodilation, and therefore there could be an increase in tissue oxygen diffusion, promoting the repair process, because the secretion of collagen by fibroblasts in the extracellular space only occurs in the presence of high rates of oxygen pressure ${ }^{1}$. However, in a meta-analysis study ${ }^{23}$, the results showed no difference when comparing the treatment of periodontal disease through SRP or in combination with lasers. These conflicting results may be due to methodological differences, mainly in relation to the protocols of laser used and to the different irradiation parameters used.

Among the limitations of the study, we can mention the fact of being carried out in animals, and it is not prudent to extrapolate the results to the human species, for further studies in the literature would be necessary.

\section{Conclusion}

Within the limits of this study, we can conclude that LLLT was effective as adjuvant treatment for SRP protecting against the occurrence of oxidative tissue damages as well as reducing alveolar bone loss in experimentally induced periodontitis simvastatinmodified rats.

\section{Conflict of interest}

The authors declare no conflict of interest in this study. 


\section{References}

1- AlGhamdi KM, Kumar A, Moussa NA. Low-level laser therapy: a useful technique for enhancing the proliferation of various cultured cells. Lasers Med Sci. 2012;27:237-49.

2- Almeida J, Ervolino E, Bonfietti LH, Novaes VC, Theodoro LH, Fernandes $L A$, et al. Adjuvant therapy with sodium alendronate for the treatment of experimental periodontitis in rats. J Periodontol. 2015;86:1166-75.

3- Baltacıoglu E, Yuva P, Aydın G, Alver A, Kahraman C, Karabulut E, et al. Lipid peroxidation levels and total oxidant/antioxidant status in serum and saliva from patients with chronic and aggressive periodontitis. Oxidative stress index: a new biomarker for periodontal disease? J Periodontol. 2014;85:1432-41.

4- Bradford MM. A rapid and sensitive method for the quantitation of microgram quantities of protein utilizing the principle of proteins-dye binding. Anal Biochem. 1976;7:248-54.

5- Cappuyns I, Cionca N, Wick P, Giannopoulou C, Mombelli A. Treatment of residual pockets with photodynamic therapy, diode laser, or deep scaling. A randomized, split-mouth controlled clinical trial. Lasers Med Sci. 2012;27:979-86.

6- Fentoğlu O, Köroğlu BK, Hiçyılmaz H, Sert T, Özdem M, Sütçü R, et al. Pro-inflammatory cytokine levels in association between periodontal disease and hyperlipidaemia. J Clin Periodontol. 2011;38:8-16.

7- Garcia VG, Fernandes LA, Macarini VC, Almeida JM, Martins TM, Bosco AF, et al. Treatment of experimental periodontal disease with antimicrobial photodynamic therapy in nicotin-modified rats. J Clin Periodontol. 2011;38:1106-14.

8- Giannelli M, Formigli L, Lorenzini L, Bani D. Combined photoablative and photodynamic diode laser therapy as an adjunct to non-surgical periodontal treatment. A randomized split-mouth clinical trial. J Clin Periodontol. 2012;39:962-70.

9- Greabu M, Totan A, Miricescu D, Radulescu R, Virlan J, Calenic B. Hydrogen sulfide, oxidative stress and periodontal diseases: a concise review. Antioxidants (Baesel). 2016;5(1). pii: E3. doi: 10.3390/ antiox5010003.

10- Houshmand B, Hassanizade R, Eslami B, Amouei S, Dashti G, Morad $\mathrm{G}$, et al. Simvastatin and lovastatin induce ectopic bone formation in rat subcutaneous tissue. J Periodontol Impl Dent. 2011;2:12-6.

11- Kara A, Akman S, Ozkanlar S, Tozoglu U, Kalkan Y, Canakci CF. Immune modulatory and antioxidant effects of melatonin in experimental periodontitis in rats. Free Radic Biol Med. 2013;55:21-6. 12- Karim S, Pratibha PK, Kamath S, Bhat GS, Kamath U, Dutta B, et al. Superoxide dismutase enzyme and thiol antioxidants in gingival crevicular fluid and saliva. Dent Res J. 2012;9:266-72.

13- Killeen AC, Rakes PA, Schmid MJ, Zhang Y, Narayana N, Marx DB, et al. Impact of local and systemic alendronate on simvastatin-induced new bone around periodontal defects. J Periodontol. 2012;83:1463-71. 14- Kohen R, Nyska A. Oxidation of biological systems: oxidative stress phenomena, antioxidants, redox reactions, and methods for their quantification. Toxicol Pathol. 2002;30:620-50.

15- Liu S, Bertl K, Sun H, Liu ZH, Andrukhov O, Rausch-Fan X. Effect of simvastatin on the osteogenetic behavior of alveolar osteoblasts and periodontal ligament cells. Human Cell. 2012;25:29-35.
16- Luan Z, Chase AJ, Newby AC. Statins inhibit secretion of metalloproteinases $-1,-2,-3$, and -9 from vascular smooth muscle cells and macrophages. Arterioscler Thromb Vasc Biol. 2003;23:769-75. 17- Meisel P, Kroemer HK, Nauck M, Holtfreter B, Kocher T. Tooth loss, periodontitis, and statins in a population-based follow-up study. J Periodontol. 2014;85:160-8

18- Monteiro LO, Macedo AP, Shimano RC, Shimano AC, Yanagihara GR, Ramos J, et al. Effect of treatment with simvastatin on bone microarchitecture of the femoral head in an osteoporosis animal model. Microsc Res Tech. 2016;79:684-90.

19- Önder C, Kurgan Ş, Altıngöz SM, Bağış N, Uyanık M, Serdar $M A$, et al. Impact of non-surgical periodontal therapy on saliva and serum levels of markers of oxidative stress. Clin Oral Investig. 2017;21(6):196-9.

20- Qi Y, Zhao T, Yan W, Xu K, Shi Z, Wang J. Mesenchymal stem cell sheet transplantation combined with locally released simvastatin enhances bone formation in a rat tibia osteotomy model. Cytotherapy. 2013;15:44-56.

21- Razzouk S. Regulatory elements and genetic variations in periodontal diseases. Arch Oral Biol. 2016;72:106-15.

22- Saglam M, Kantarci A, Dundar N, Hakki SS. Clinical and biochemical effects of diode laser as an adjunct to nonsurgical treatment of chronic periodontitis: a randomized, controlled clinical trial. Lasers Med Sci. 2014;29:37-46.

23- Sgolastra F, Severino M, Gatto R, Monaco A. Effectiveness of diode laser as adjunctive therapy to scaling root planning in the treatment of chronic periodontitis: a meta-analysis. Lasers Med Sci. 2013;28:1393-402.

24- Singh N, Chander Narula S, Kumar Sharma R, Tewari S, Kumar Sehgal P. Vitamin E supplementation, superoxide dismutase status, and outcome of scaling and root planing in patients with chronic periodontitis: a randomized clinical trial. J Periodontol. 2014;85:242-9. 25- Subramanian S, Emami H, Vucic E, Singh P, Vijayakumar J, Fifer $\mathrm{KM}$, et al. High-dose atorvastatin reduces periodontal inflammation: a novel pleiotropic effect of statins. J Am Coll Cardiol. 2013;62:2382-91. 26- Surve SM, Acharya AB, Thakur SL. Efficacy of subgingivally delivered atorvastatin and simvastatin as an adjunct to scaling and root planing. Drug Metab Pers Ther. 2015;30:263-9.

27- Tebloeva LM, Gurevich KG. Osteoimmunnology and periodontitis. Patol Fiziol Eksp Ter. 2014;3:67-72.

28- Tomofuji T, Ekuni D, Irie K, Azuma T, Tamaki N, Maruyama T, et al. Relationships between periodontal inflammation, lipid peroxide and oxidative damage of multiple organs in rats. Biomedical Research. 2011;32:343-9.

29- Tonguç MÖ, Özturk O, Sütçü R, Ceyhan BM, Kılınç G, Sönmez Y, et al. The impact of smoking status on antioxidant enzyme activity and malondialdehyde levels in chronic periodontitis. J Periodontol. 2011;82:1320-8.

30- Tymkiw KD, Thunell DH, Johnson GK, Joly S, Burnell KK, Cavanaugh $\mathrm{JE}$, et al. Influence of smoking on gingival crevicular fluid cytokines in severe chronic periodontitis. J Clin Periodontol. 2011;38:219-28. 